\title{
Transduction du signal mitogène, cytosquelette et petites protéines $G$ : vers un réseau de protéines GAP?
}

Philippe Fort Sylvie Vincent

\section{ADRESSE}

Ph. Fort : chargé de recherches au Cnrs. S. Vincent : boursière MRT. URA Cnrs 1191, génétique moléculaire, universitć Montpellier II CP 101, place E.-Bataillon, 34095 Montpellier Cedex 5, France.

$\mathrm{m} / \mathrm{s} n^{\circ} 1$ vol. 9 , janvier 93

D'importants progrès ont été faits au cours de ces derniers mois dans la détermination de la fonction cellulaire des petites GTPases de la famille Rho. Il vient en effet d'être montré que deux membres de cette famille (Rac et Rho) sont impliqués dans le contrôle de la réorganisation des réseaux d'actine cytosquelettique provoquée par les facteurs de croissance. En outre, la découverte de plusieurs facteurs multifonctionnels, possédant un domaine GAP capable de moduler l'activité des protéines Rho, ainsi que des activités extrêmement diverses telles que sérine kinase ou répresseur transcriptionnel, suggère que les protéines de la famille Rho sont réglées de façon concertée avec les protéines Ras, et interviennent dans la transduction des signaux mitogènes. La traque des facteurs GAP ainsi que la détermination de leurs cibles permettront-elles d'obtenir une vision intégrée des processus de division et de réorganisation de l'architecture cellulaire?

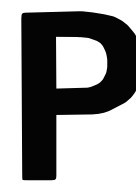

armi les familles de protéines caractérisées chez les eucaryotes, la superfamille des petites GTPases apparentées à Ras a connu une expansion impressionnante au cours de ces dix dernières années. On recense actuellement une cinquantaine de membres, regroupés en 5 sous-familles Ras, Rho, Rab, ARF (ADP ribosylation factor) et TC4 [1-3]. Si les critères adoptés pour effectuer cette classification sont essentiellement structuraux (similarité de séquence), on constate cependant une excellente correspondance avec les caractéristiques fonctionnelles des protéines regroupées. Il est en effet solidement établi que les membres de la famille Ras sont des composants essentiels des voies de transduction des signaux de prolifération et de différenciation cellulaires, tandis que les membres des familles Rab et ARF interviennent dans le transport des vésicules entre les compartiments intracellulaires [4]. En revanche, la situation de la famille Rho est plus complexe, puisqu'elle présente une ramification interne : un premier regroupement 


\section{RÉFÉRENCES}

1. Valencia A, Chardin P, Wittinghof er A, Sander C. The ras family : cvolutionary trec and role of conserved aminoacids. Biochemistry $1991 ; 19$ : 4637-48.

2. Chardin P. Small G'TP-binding protcins of the ras family: a conserved functional mechanism ? Cancer Cells 1991; 3 : 117-26.

3. Hall A. The cellular functions of small GTP-binding protcins. Science $1990 ; 249$ : $635-40$

4. Goud B. L.c transport vćsiculaire des cellules cucaryotes est contrôlć par des G'ГPascs. médecine/sciences $1992 ; 8$ : 326-34.

5. Vincent $\mathrm{S}$, Jeantcur Ph, Fort Ph. Growth-regulated expression of rhoG, a new member of the ras homolog gene family. Mol Cell Biol 1992 ; 12 : 3138-48.

6. Chardin P, Boquet P, Madaulc P, Popoff MR, Rubin EJ, Gill DM. The mammalian $\mathrm{G}$ protein rhoC is ADP-ribosylated by Clostridium botulinum cxocnzyme C3 and affects actin microfilaments in Vero cells. EMBO J $1989 ; 8: 1087-92$.

7. Paterson H, Sclf AJ, Garrett MD, Just I, Aktorics K, Hall A. Microinjection of recombinant $\mathrm{p} 21$ tho induces rapid changes in cell morphology. J Cell Biol $1990 ; 111$ : 1001-07.

8. Abo A, Pick E, Hall A, Totty N, Tcahan CG, Segal AW. Activation of the NADPH oxidase involves the small GTPbinding protcin p21 racl. Nature 1991; 353 : $668-70$.

9. Ridlcy AJ, Hall A. The small G'TPbinding protein rho regulates the assembly of focal adhesions and actin stress fibers in response to growth factors. Cell $1992 ; 70$ : $389-99$

10. Ridley AJ, Paterson HF, Johnston CL, Dicckmann D, Hall A. 'The small G'TP. binding protein rac regulates growth factorinduced membranc ruffling. Cell 1992; 70 : 401-10.

11. Kolega J. Effects of mechanical tension on protusive activity and microfilament and intermediate filament organization in an cpidermal cpithelium moving in culture. $J$ Cell Biol 1986 ; 102 : 1400-11.

12. Stcincrt PM, Roop DR. Molccular and cellular biology of intermediatc filaments. Annu Rev Biochem 1988; 57 : 593-626.

13. Karsenti E, Maro B. Centrosomes and the spatial of microtubules in animal cells. Trends Biochem Sci 1986; 11: 460-3.

14. Bar-Sagi D, Feramisco JR. Induction of membrane ruffling and fluid-phase pinocytosis in quicscent fibroblasts by ras pro-

englobe RhoA, B et $\mathrm{C}$, plus un nouveau membre RhoD (T. Hunter, communication personnelle), le second rassemble Rac1 et 2, TC10, CDC42/G25K et rhoG, récemment caractérisé [5]. Le premier indice sur la fonction des protéines RhoA, B et $\mathrm{C}$ a été obtenu en inhibant lcur activité par ADP-ribosylation de leur résidu asparagine (position 41), grâce à l'utilisation de l'exoenzyme C3 transférasc produite par Clostridium botulinum. L'introduction de cette toxine C3 dans différents types cellulaires provoque une désorganisation importante du cytosquelette, et en particulier une dépolymérisation des filaments d'actine [6]. Parallèlement, la surexpression de RhoA et C par micro-injection de protéines recombinantes ou de vecteurs d'expression induit la formation de filaments d'actine [7]. La fonction des autres protéines de la famille Rho est moins claire : on a d'abord montré que les protéines Rac pcuvent activer une NADPH oxydase et stimuler la production de superoxyde en systèmc reconstitué, à partir d'extraits de phagocytes [8]. Ccpendant, si le gènc rac-2 est exprimé spécifiquement dans la lignéc hématopoïétique, rac-1 présente une expression constitutive, qui laisse supposer une fonction différente dans les autres types cellulaires. CDC42Sc, homologue chez la levure dc CDC42Hs, est impliqué dans les mécanismes de polarité cellulaire, elle-même corrélée à la distribution spatiale des filaments d'actine. Les fonctions des deux derniers membres, RhoG et TC10, sont inconnues. Toutefois, des résultats préliminaires de surexpression obtenus dans notre laboratoire indiquent que RhoG, principalement exprimé dans les tissus pulmonaire et placentaire, participe également à l'architecture générale du cytosquelette.

Les protéines de la superfamille Ras peuvent être présentes sous une forme liée au GTP ou liée au GDP. Le passage de l'une à l'autre fait intervenir, d'une part, une stimulation de leur activité GTPasique intrinsèque (sens GTP vers GDP) par une protéine GAP (GTPase activating protein) et, d'autre part, un processus d'échange (sens GDP vers GTP), pouvant être réglé négativement par une protéine GDI (GDP dissociation inhibitor) ou positivement par une protéine GDS (GDP dissociation stimulator). D'une manière générale, on constate que les protéines GAP sont plutôt spécifiques d'une famille; cela est dû au fait qu'elles reconnaissent la portion $\mathrm{NH}_{2}$-terminale très conservée entre lcs membres d'une même famille. En revanche, les facteurs d'échange, qui interagissent avec la région $\mathrm{COOH}$ terminale, très hétérogène d'un membre à l'autre, présentent davantage de spécificité individuelle. Au cours des derniers mois, divers travaux ont nettement modifié l'éclairage du paysage des petites protéines $G$, en donnant plus de lumière sur le rôle des protéines de la famille Rho, ainsi que sur la naturc des régulateurs des familles Rho et Ras.

\section{Protéines Rho et polymérisation des fibres d'actine}

Anne Ridley, Alan Hall et leurs collègucs viennent de publicr des résultats montrant que les protéines RhoA et Rac1 sont impliquécs dans la réorganisation des fibres d'actine dc plusieurs structures cellulaires $[9,10]$. Rappelons brièvement que lc cytosquelette est un réseau de fibres protéiques développé dans l'ensemble du cytoplasme, et constitué de trois types de filaments interconnectés, les filaments intermédiaircs, les microtubules et les microfilaments (pour revue, voir [11-13]).

Contrairement aux microtubules ct aux microfilaments, les filaments intcrmédiaires sont des structurcs cellulaires stables ct rigidcs. Généralcment disposés en faisceaux ou en réseaux, ils permettent d'absorber les tensions mécaniques exercécs par les mouvements des tissus : ainsi, les kératincs assurent la rigidité des cellules épithéliales, tandis que la desmine permet de maintenir la cohésion des cellules musculaires lisses et striées pendant la contraction. Les microtubules (sortes de cordcs creuses de dimères de tubuline $\alpha$ et $\beta$ ) interviennent dans la dynamique cellulaire dans diverses structures. Le dénominatcur commun cst cn fait représenté par les centrioles, minicylindres de microtubules, à partir desquels de longues fibres vont se développer par un processus dc poly- 
mérisation qui requicrt du GTP. Chez les eucaryotes supérieurs, les centrioles, localisés dans le centrosome, sont à l'originc des faisceaux de microtubules périnucléaires de la cellule intcrphasique, tandis que chez les ciliés et flagellés cucaryotes, les centrioles du corps basal sont responsables de la formation des flagelles. Dans les deux situations, les cellules en cours de division réarrangent leurs centrioles en centrosomes polaires (spindle poles) d'où partent les microtubules du fuseau mitotique. Les microfilaments, qui représentent cn fait le système contractile des cellules, sont constitués de filins de molécules d'actinc $\beta$ ou $\gamma$ polymérisécs. On trouve également des fibres d'actine dans les muscles squelettiques, sous une forme particulièrement bien développée et organisée, en association avec des cordes de myosine. Dans les cellules non musculaircs, les fibres d'actine, moins denses ct très instables, participent à dc nombreuscs fonctions cellulaircs qui requièrent toutes d'importantes déformations membranaires. Ces fibres sont polymérisées dans lc cortex membranaire à partir dc différentes structures ccllulaircs (microvillosités des ccllules intestinales, cônes de croissance axonaux, pscudopodes de macrophages, anncaux contractiles de cellules en télophase...), ct forment des conncctions dynamiques entre ces structures membranaires, et des interconnexions avec le réscau microtubulaire périnucléairc. Dans les cellules de typc fibroblastique, on trouve les filaments d'actine, associés à d'autres protéines, dans les excroissances membranaires (membrane ruffling), dans les microspicules et les lamellipodes (ruffles) et dans les fibres de tension (stress fibers), ancrées aux plaques d'adhérence (focal adhesions) par un complexe protéique dans lequel on retrouve entre autres la taline et la vinculine (figure 1). La plasticité de ces structures membranaires dépend de l'équilibre entre la polymérisation et la dépolymérisation des fibres d'actine, et intervient dans des processus aussi variés que le déplacement cellulaire (cytokinèse), la formation de vésicules (pinocytosc, endocytose) et la division cellulaire (forme arrondie des cellules).

Les résultats du groupe d'Alan Hall établissent clairement qu'une réorga$\mathrm{m} / \mathrm{s} n^{\circ} 1$ vol. 9, janvier 93

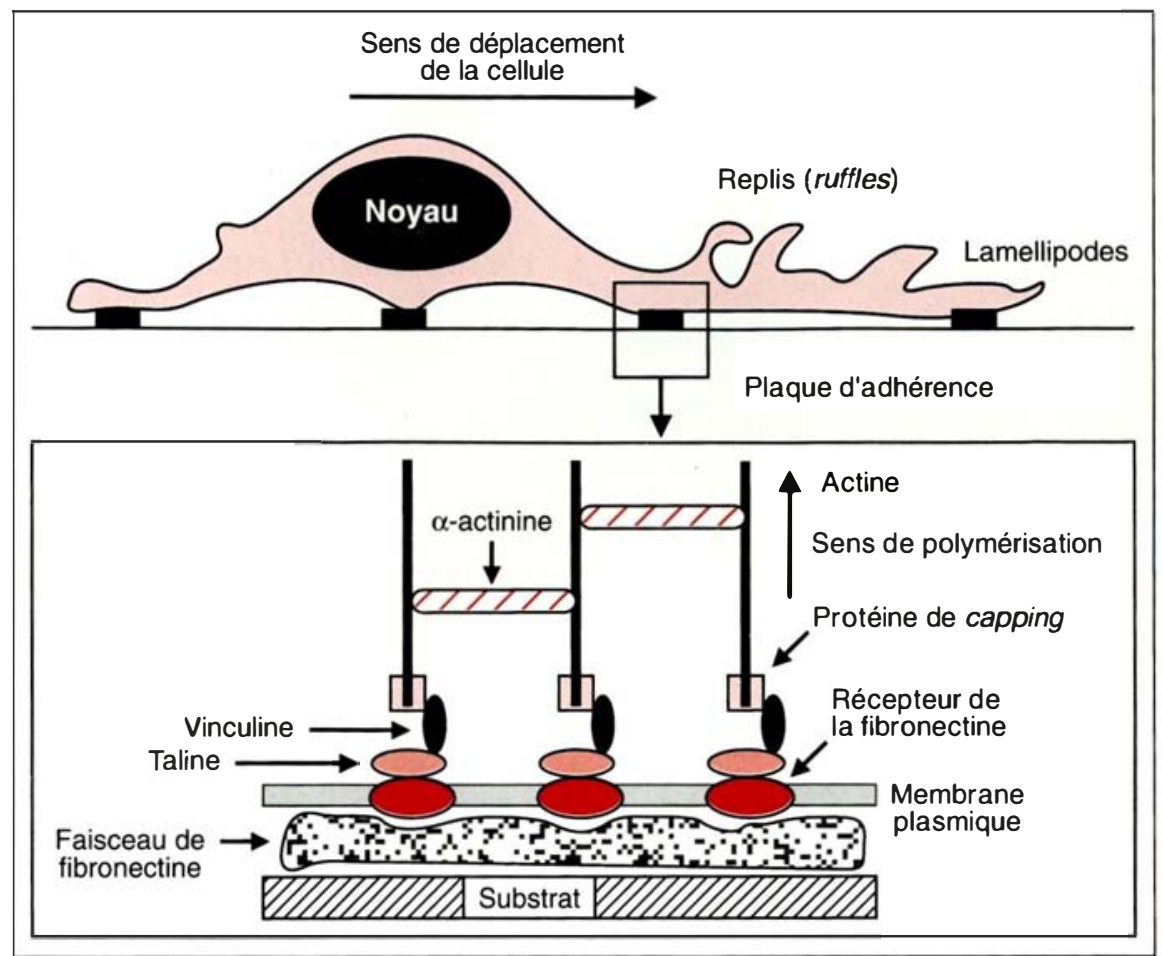

Figure 1. Représentation schématique d'une cellule fibroblastique en déplacement. La cellule émet des prolongements membranaires (lamellipodes) dans le sens de la progression, à la surface desquels les plaques d'adhérence sont formées (encadré). Les fibres d'actine sont polymérisées à partir des plaques $d^{\prime}$ adhérence, et rejoignent le réseau de micro-tubules périnucléaires ou d'autres plaques d'adhérence. La tension des fibres dans une direction permet le déplacement cellulaire.

nisation des fibres d'actinc au niveau des fibres de tension et des plaques d'adhérence ainsi que la formation de replis membranaircs interviennent dans les minutes qui suivent l'addition de facteurs de croissance, et nécessitent l'activité des protéines Rho (figure 2). L'intensité et la vitesse auxquelles ccs remaniements apparaissent dépendent de la nature de l'agent utilisé pour la stimulation. Deux voies de transduction ont été mises en évidence : la première fait intervenir l'acide lysophosphatidique (LPA), un phospholipide présent dans le sérum de veau fœtal, connu pour être un puissant stimulateur de l'agrégation plaquettaire, ainsi qu'un agent mitogène pour un certain nombre de types cellulaires. La deuxième, moins efficace, passe par la voic des récepteurs de type tyrosinc kinase, tels que le PDGF et l'EGF. Lorsque l'activité des protéines Rho (A, B ou C) cst inhibée (par micro-injection de toxinc botulinique C3), la formation des fibres de tension et des plaques d'adhérence est bloquée, quelle que soit la voie de stimulation choisic, sans que la formation de replis membranaircs ne soit altérée. Si l'on inhibe l'activité Rac endogène par micro-injection d'une protéine mutante inhibitricc dominante, la formation de fibres de tension est bloquéc uniquement si le signal transmis passe par la voie des récepteurs tyrosine kinase. En revanche, on n'observe plus de replis membranaires quelle que soit la voie empruntée. Ces observations en "négatif " sont confirmées par des analyses "positives " : la micro-injection de protéines dans des cellules au repos entraîne l'apparition de fibres de tension et de replis membranaires dans le cas de Rac, et seulement des fibres de tension dans le cas de Rho. En outre la micro-injection de $\mathrm{Rac}$ couplée à l'inhibition de Rho active unique- 


\section{RÉFÉRENCES}

15. Reibel L, Dorseuil O, Stancou R, Bertoglio J, Gacon G. A hemopoictic spccific genc encoding a small GTP binding protein is overexpressed during $\mathrm{T}$-cell activation. Biochem Biophys Res Commun 1991; 175 : 451-8.

16. Jahner $\mathrm{D}$, Hunter $\mathrm{T}$. The ras-related genc $r h o B$ is an immediate-carly gene inducible by $\mathrm{v}$-f ps, epidermal growth factor, and platelet-derived growth factor in rat fibroblasts. Mol Cell Biol 1991; 11 : 3682-90.

17. Hart MJ, Eva A, Evans T, Aaronson SA, Cerione RA. Catalysis of guanine nuclcotide exchange on the $\mathrm{CDC} 42 \mathrm{Hs}$ protein by the dbl oncogene product. Nature $1991 ; 354$ : 311-4.

18. Dieckmann D, Brill S, Garrett MD, et al. $B c r$ encodes a GTPase-activating protein for p21 ras. Nature 1991; 351: 400-2.

19. Cicchetti P, Mayer BJ, Thiel G, Baltimorc D. Identification of a protein that binds to the $\mathrm{SH} 3$ region of $\mathrm{Abl}$ and is similar to Bcr and GAP-rho. Science $1992 ; 257$ : 803-6.

20. Filhol O, Cochet C. Le transfert des signaux mitogćniques : unc affairc de particulcs. médecine/sciences $1990 ; 6: 980-4$.

21. Koch CA, Anderson D, Moran MF, Ellis C, Pawson T. SH2 and SH3 domains : elements that control interactions of cytoplasmic signalling protcins. Science 1991; 252 : 668-74.

22. Maru Y, Witte ON. The $B c r$ gene encodes a novel serine/threonine kinase activity within a single exon. Cell 1991; 67 . 459-68.

23. Shou C, Farnsworth CL, Neel BG, Fcig LA. Molecular cloning of cDNAs encoding a guanine-nucleotide-releasing factor for Ras p21. Nature 1992 ; 358 : 351-4.

24. Jones S, Vignais ML, Broach JR. The CDC25 protein of Saccharomyces cerevisiae promotes exchange of guanine nucleotides bound to Ras. Mol Cell Biol 1991; 11 : 2641-6.

25. Simon MA, Bowtell DDL, Dodson GS, Laverty TR, Rubin GM. Ras1 and a putative guaninc nuclcotide exchange factor perform crucial steps in signalling by the sevenless protein tyrosine kinasc. Cell 1991; 67 : 701-16.

26. Galland F, Birnbaum D. Le protooncogene $m c f 2 / d b l$ et les facteurs d'ćchange GDP-GTP. médecine/sciences $1992 ; 8$ : 819-26.

27. Settleman J, Narasimhan V, Foster LC, Weinberg RA. Molccular cloning of cDNAs encoding the GAP-associated protcin p190 : implications for a signalling pathway from ment la formation des replis, suggérant un rôle régulateur des protéines Rac sur l'activité des protéines Rho.

\section{Rôle de Ras dans la réorganisation du cytosquelette}

Ces observations confirment l'implication de la famille Rho dans l'organisation générale du cytosquelette, tout en permettant de préciser les relations fonctionnelles entre les protéines Rho et Rac. En outre, elles impliquent plus nettement la famille Rho dans les réorganisations des structures membranaires ct du cytosquelette associées à la prolifération cellulaire. En effet, une cellule fibroblastique en phase de repos (après retrait des facteurs de croissance) montre des densités de fibres de tension et de lamellipodes très faibles. L'entrée en phase G1 se caractérise par une forte augmentation du nombre de fibres de tension et une densité accrue d'excroissances membranaires. Au cours de la progression vers la synthèse d'ADN (phase $S$ ), ces structures disparaissent au profit de microvillosités, pendant que la cellule adopte une formc arrondie, nécessaire pour la poursuite du cycle (le maintien forcé d'une morphologie étalée empêche les cellules d'entrer en phase S). On peut donc envisager qu'il existe un dialogue entre les protéines Ras et Rho, assurant la bonne coordination des programmes mis en place.

En fait, il avait déjà été constaté dès 1986 que la micro-injection de protéines Ras activées induisait la formation de structures membranaires, ainsi qu'une augmentation de la pinocytose [14], d'une manière tout à fait similaire à celles induites par Rac. La relation entre Ras et Rac a donc été testée : la micro-injection de protéine Ras activée n'induit plus de remaniements membranaires ni de formation de fibres de tension si l'on co-injecte un inhibiteur de Rac. Cependant, si l'on bloque l'activité Ras endogène, l'induction des réorganisations membranaires en réponse aux facteurs de croissance est toujours maintenue. Ces résultats suggèrent l'existence de deux voies de signalisation couplant facteurs de croissance et activité Rac, dont l'une est indépendante de Ras (figure 2).

\section{Protéines Rho et transformation cellulaire}

A la lumière des données récentes, il apparaît vraisemblable que l'activité des protéines Rho est requise pendant la prolifération cellulaire. On sait en eff et que plusieurs gènes codant pour des protéines Rho sont induits par des agents mitogènes: le niveau d'expression de rac-2 augmente fortement dans des lignées lymphocytaires $\mathrm{T}$ stimulées en croissance [15], tandis que $r h o B$ et $r h o G$ appartiennent respectivement aux gènes de réponse précocc et tardive à un large spectre de facteurs de croissance de cellules fibroblastiques $[5,16]$. On dispose en outre d'informations qui impliquent plus directement les protéines Rho dans les processus de transformation cellulaire. Ainsi, des lignées fibroblastiques transfectées avec une version activée de rhoA présentent des phénotypes partiellement transformés, et peuvent former des tumeurs dans l'animal après implantation souscutanéc. Par ailleurs, deux protéines régulatrices de la famille Rho présentent des versions oncogéniques caractéristiques de certaines leucémies myéloïdes et lymphoïdes. Il s'agit de Dbl [17], un facteur d'échange spécifique de CDC42Hs, et de Bcr [18], qui possède dans sa région $\mathrm{COOH}$ terminale un domaine GAP capable d'accélérer les activités GTPasiques des protéines Rac et $\mathrm{CDC} 42 \mathrm{Hs}$, et non celles de RhoA, B et C. Un troisième exemple est celui de l'oncogène vav, qui code pour une protéine possédant des domaines similaires à $\mathrm{Dbl}$ et $\mathrm{Bcr}$, et qui pourrait être également un facteur régulateur de l'activité de protéines Rho. Tous ces éléments suggèrent que si les protéines Rho ne sont pas stricto sensu des protéines oncogéniques, elles participent néanmoins à des voies métaboliques dont le dérèglement favorise la transformation cellulaire.

\section{$G A P^{\text {tho }}$ et tyrosine kinases de type non récepteur}

Les protéines codées par les protooncogènes $c$-src ct $c$-abl possèdent des domaines SH2 et SH3 (Src homology) 


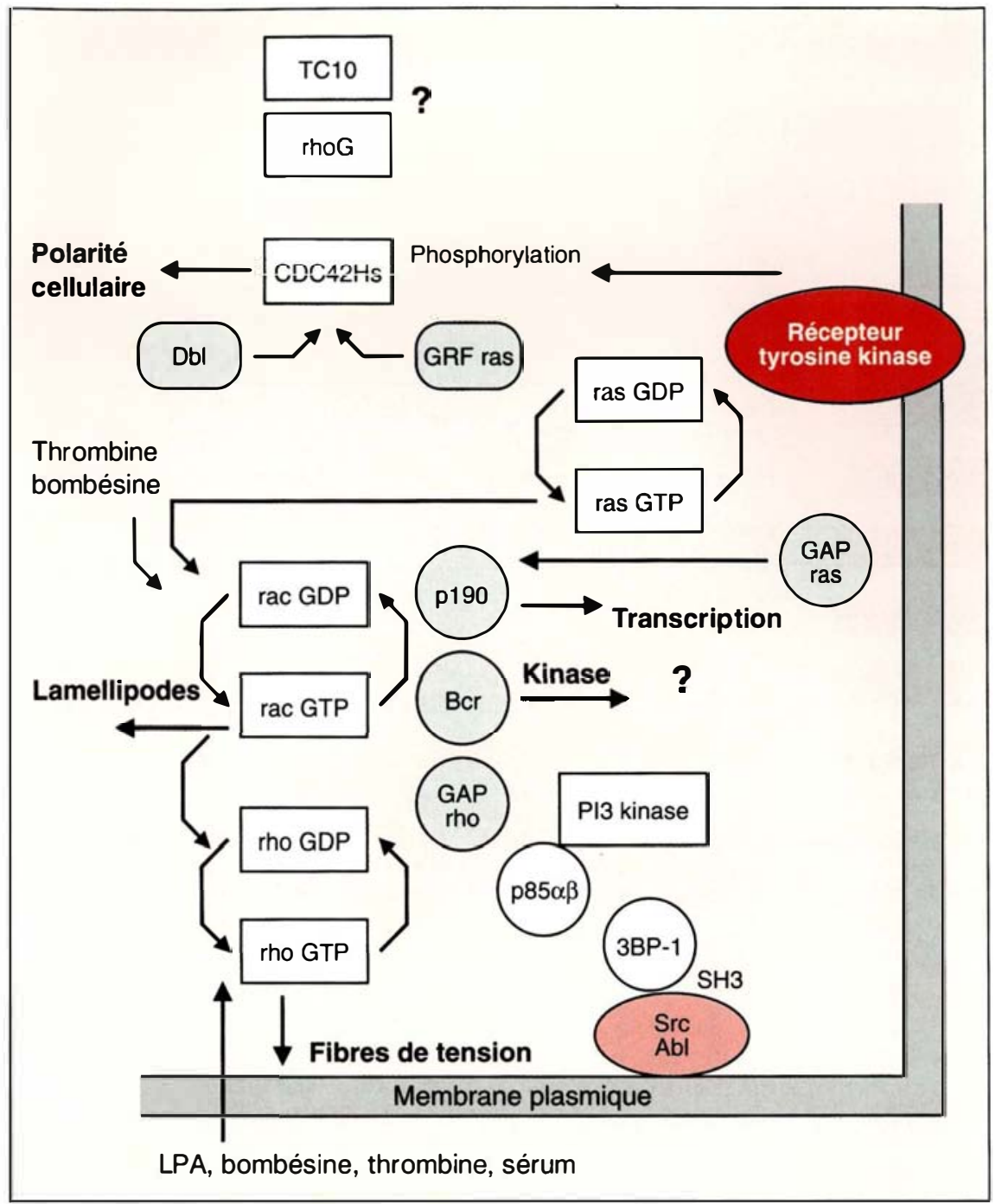

Figure 2. Voies métaboliques dans lesquelles les membres de la famille Rho sont susceptibles d'agir. La structure générale des protéines GAP dont l'activité a été testée (cercles grisés) ou non testée (cercles blancs), ainsi que des facteurs d'échanges (rectangles ovalisés et grisés) est représentée sur la figure 2. LPA: acide lysophophatidique. PI 3 : phosphatidyl-inositol-3. SH3 : Domaine 3 d'homologie avec Src.

impliqués pour les uns dans la fixation de résidus phosphotyrosine, pour les autres dans la liaison aux fibres d'actine du cytosquelette. La délétion des domaines SH3 est suffisante pour activer le caractère transformant de ces deux protéines. Src et Abl appartiennent à la famille des tyrosine kinases membranaires, mais sans domaine récepteur. Src est particulièrement concentrée au niveau des plaques d'adhérence, d'où partent les fibres de tension, et son activation oncogénique engendre rapidement la formation de replis membranaires. Le

$\mathrm{m} / \mathrm{s} n^{\circ} 1$ vol. 9 , janvier 93 directement certaines protéines Rho dans une voie altcrne de transduction de signal. Deux protéines supplémentaires, p85 $\alpha$ et p85 $\beta$, présentent une région $\mathrm{GAP}^{\text {rho }}$ analogue à $\mathrm{Bcr}$, ainsi que des domaines $\mathrm{SH} 2$ et $\mathrm{SH} 3$ [21]. Elles sont chacune une sous-unité régulatrice du complexe enzymatique de la phosphatidylinositol (PI) 3 kinase, qui conduit à la synthèse de PI 3 phosphate et dérivés, et dont l'implication dans la transduction du signal mitogène est de plus en plus suspectée. Ces protéines présentent une structure très voisine de la GAPras, qui contient également des domaines $\mathrm{SH} 2$ et $\mathrm{SH} 3$. Curieusement, certaines translocations dans les leucémies myéloïdes chroniques ou lymphoïdes aiguës impliquent les gènes $c$ - $a b l$ et $b c r$, et conduisent à la présence de protéines chimères $\mathrm{Bcr} / \mathrm{Abl}$ dont l'activité tyrosine kinase est déréglée. Or, il est maintenant établi que le domaine Bcr impliqué contient un nouveau type d'activité sérine kinase [22]. Est-ce cette nouvelle activité, et non celle portée par $\mathrm{Abl}$, qui confère à la protéine chimère ses propriétés transformantes ? Plus encore, l'événement réciproque de la translocation est susceptible de produire une protéine chimère $\mathrm{Abl} / \mathrm{Bcr}$, dont le domaine GAP serait fusionné à la région $\mathrm{NH}_{2}$-terminale de $\mathrm{Abl}$, où sont localisés les domaines $\mathrm{SH} 2$ et $\mathrm{SH} 3$. Une telle protéine présenterait donc une structure très voisine de la GAPras et des p85 $\alpha$ et $\beta$, et pourrait provoquer des interférences entre les voies métaboliques dans lesquelles $\mathrm{R}$ as et Rho sont impliquées.

\section{Régulation concertée entre les protéines Rho et Ras}

groupe de $\mathrm{D}$. Baltimore a récemment identifié une nouvelle protéine (3BP-1) capable de fixer fortement des domaines SH3 de Src et Abl, et présentant des domaines GAP homologues à celui de Bcr [19]. 3BP-1 a donc la potentialité de coupler une activité GTPasique spécifique de membres de la famille Rho et une protéine tyrosine kinase de type non récepteur. Une telle association serait structuralement similaire au complexe métabolique récepteur tyrosine kinase/phospholipase C/GAP ras/p21 ras [20], et pourrait donc impliquer
Un facteur d'échange GDP/GTP de p21 ras a été récemment caractérisé par le groupe de Larry Feig [23]. Il s'agit d'une protéine de $140 \mathrm{kDa}$ dénommée GRF ras (guanine nucleoside releasing factor for Ras $p 21$ ), dont la région carboxy-terminale est similaire à celle de la protéine de levure CDC25, facteur d'échange de Ras [24], et à celle de la protéine de drosophile Sos, impliquée dans la voie de signalisation du récepteur tyrosine kinase sevenless ([23], $\mathrm{m} / \mathrm{s} n^{\circ} 5$, vol. 8, p. 471 et $n^{\circ} 10$, vol. 8, p. 1097). 


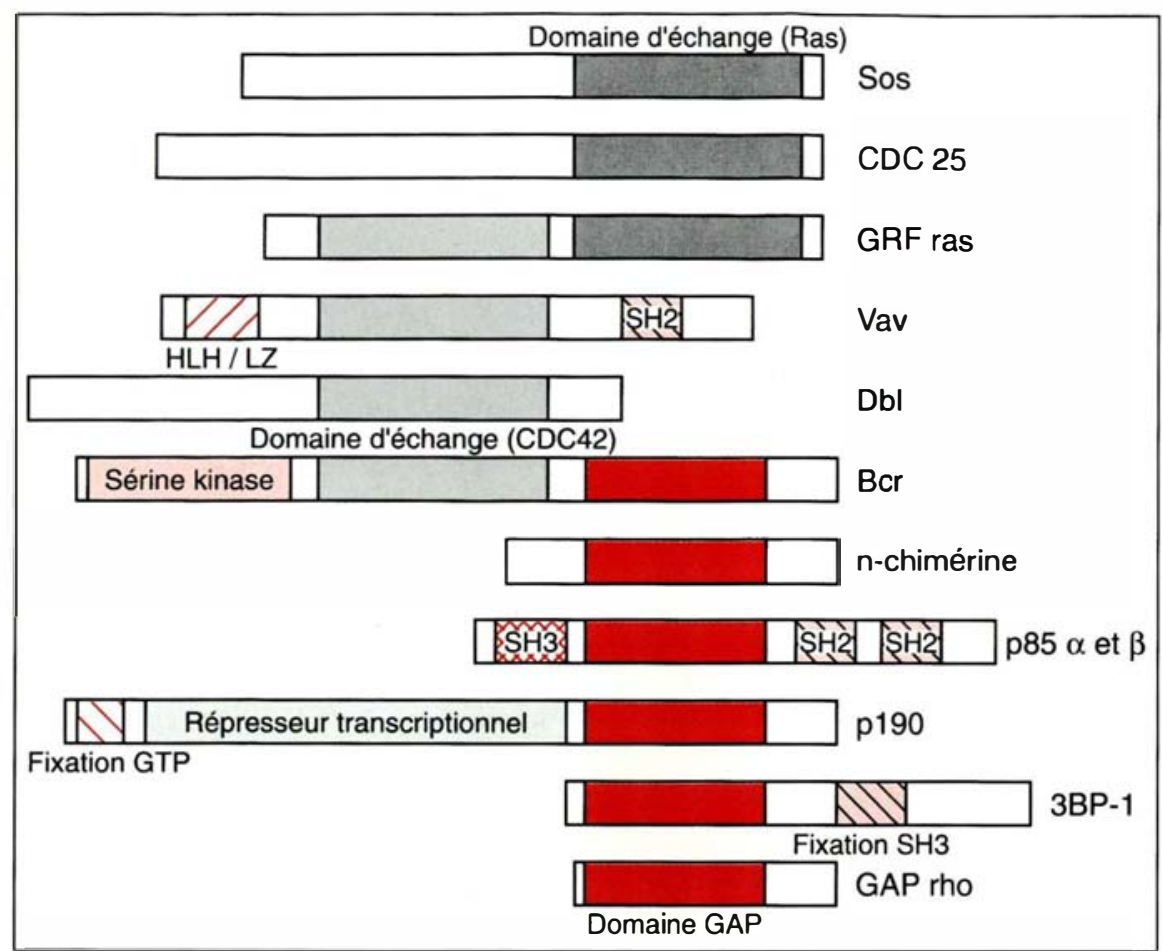

Figure 3. Structure schématisée des protéines régulatrices potentielles de la famille Rho. L'activité GAPrho, portée par la région figurée en noir, a été testée par GAPrho, Bcr, n-chimérine et p190. Le domaine d'homologie entre GRFras, Dbl et Bcr est figuré en gris. SH2 et SH3 : Domaines 2 et 3 d'homologie avec Src. HLH/LZ: Domaines hélice/tour/hélice et leucine zipper.

Cependant, une portion de 230 acides aminés dans la région aminoterminalc présente unc grandc similarité avec Bcr, Dbl et Vav (figure 3) [26]. Bien qu'il n'ait pas été encore prouvé que la p140 possède unc activité d'échange sur $\mathrm{CDC} 42 \mathrm{Hs}$, cela suggère une interférence entre les régulations de Ras et $\mathrm{CDC} 42 \mathrm{Hs}$. Et l'histoire ne s'arrête pas là : la GAP $^{\text {ras }}$ est capable de former rapidement des complexcs de hauts poids moléculaires dans des ccllules traitées par l'EGF. L'un des complexcs fait intervenir une protéinc p190, l'autre une protéine fortement phosphorylée, pp62. Ces deux protéines ont été récemment caractérisćes [27, 28]. Il ressort quc pp62 possèdc un domaine de fixation à l'ARN similaire à celui d'une protéine localisée probablement dans les hnRNP. L'autre protéine, p190, intrigue encore davantage : elle contient dans sa région $\mathrm{NH}_{2}$ un domaine de fixation du GTP, dans sa région $\mathrm{COOH}$ un domaine GAP similaire à $\mathrm{Bcr}$, et dans sa région centrale, un domaine de 778 acides ami- répresseur transcriptionnel du gènc du récepteur des glucocorticoïdes (figure 3). On sait maintenant que p190 et RhoGAP sont actives sur Rho (A, B et C), Rac $(1,2)$ et $\mathrm{CDC} 42 \mathrm{Hs}$, tandis que Bcr n'est actif que sur Rac $(1,2)$ et $\mathrm{CDC} 42 \mathrm{Hs}$ ([29] et A. Hall, communication personnelle). Toutefois, bien qu'aucune donnće ne permet actuellement de définir le rôle exact de cette p190 et la façon dont les protéines Rho interviennent, il s'agit là d'une pièce manquante du puzzle, assurant le licn entre les événements biochimiques cytoplasmiques engendrés par $\mathrm{R} a s$ et les régulations géniques nucléaires. Une autre pièce pourrait être apportée par la caractérisation récente d'une protéine de levure (RhoNUC) présentant toutes les caractéristiques d'une protéine Rho dans sa région amino-terminale, et d'une endonucléase dans sa région carboxy-terminale [30], cette dernic̀re activité étant impliquée dans les mécanismes de recombinaison et de réparation de l'ADN.

En conclusion, les résultats récents concernant les petites protéincs $G$ de la famille Rho font clairement participer ces dernières au contrôlc de la polymérisation de l'actinc dans différents types de structures cellulaircs en réponse à divers stimuli mitogènes. On consolide donc le schéma de départ, reliant les protéincs Ras à la transduction du signal mitogène ct les protéines Rho au contrôle de la morphologie cellulaire. Cependant, la démarcation entre ces deux aspects reste passablement floue : l'activation via les récepteurs tyrosine kinase peut conduire à une stimulation mitogène dc cellules fibroblastiques (cas le plus fréquemment décrit dans la littérature) ou à l'arrêt de la division ccllulaire (cas du NGF sur lcs cellules de phéochromocytome PC12). Or, dans les deux cas, l'interaction ligand/réceptcur est relayće par Ras de façon très semblable, sinon identique $\left(m / s \quad n^{\circ} 5\right.$, vol. $8, \quad p .471$ et $n^{\circ} 10$, vol. 8, p. 1097). De même, le remaniement du cytosquclettc intervient certes dans les processus de prolifération, mais aussi au cours de phénomènes de migration cellulaire non associés à la division. S'il scmble toutcfois que la famille Ras, cu ćgard à ses potentialités oncogéniques, a unc position hićrarchique dominante sur la famillc Rho au cours du cycle cellulairc (figure 2), on peut néanmoins spéculer sur la nécessité d'une activation coordonnée des deux voies Rho et Ras pour permettre la mise en place correcte du programme de division cellulaire. Une spéculation étayće par l'identification de protéines bifonctionnelles présentant des domaines Rho ou RhoGAP et des domaines d'activité nucléaire

\section{RÉFÉRENCES}

28. Wong W, Müller O, Clark P, et al. Molecular cloning and nuclcic acid binding properties of the GAP-associated tyrosine phosphoprotein p62. Cell $1992 ; 69$ : 551-8.

29. Settleman J, Albright CF, Foster I.C, Wcinberg RA. Association between GTPase activators for Rho and Ras familics. Nature 1992 ; 359 : 153-4.

30. Chow TYK, Pcrkins AL, Resnick MA Ycast RNC1 cncodes a chimcric protcin, RhoNUC, with a human rho motif and dcoxyribonucleasc activity. Nucleic Acids Res 1992; 20 : 5215-21. 


\section{Summary}

Signal transduction, cytoskcleton, and small GTPases : the GAP connection

Since several years, small GTPbinding proteins of the Rho family are thought to be involved in cytoskeleton organization. Recent data evidence that two of them are directly responsible for actin polymerisation. RhoA mediates actin stress fiber formation in response to growth factors, while Rac controls RhoA activity as well as the formation of actin filaments in membrane ruffles. These studies thus identify $\mathrm{Rac}$ as a main regulatory factor that controls the actin network in response to extracellular factors. Like other Ras like proteins, Rho activity is down regulated by GAP (GTPase Activating Protein), and up regulated by GRF (Guanine Releasing Factor). Several cDNAs coding for proteins with GAPrho or GRF domains have been recently isolated. The story gets much more complicated by the fact that they also contain other domains as diverse as $\mathrm{SH} 2$ or $\mathrm{SH}$, serine kinase, or transcriptional repressor regions. Might this new class of proteins constitute the missing link between the metabolic pathways signalling cellular division and cell shape reorganization? No doubt that this question will be soon answered.

TIRÉS A PART

Ph. Fort.

$\mathrm{m} / \mathrm{s} n^{\circ} 1$ vol. 9, janvier 93
Une journée Recherche dans le cadre d'EUROCANCER 93 se tiendra à Paris le 27 avril 1993, sous la présidence de P. Tambourin et $M$. Boiron, sur le thème "De la recherche oncologique à l'innovation thérapeutique " avec. entre autres, le patronage de I'INSSERM.

\section{Programme}

- Introduction et présentation de la journée (P. Tambourin, Paris) ;

- Génétique des antigènes de rejet tumoral : nouvelles perspectives pour l'immunothérapie spécifique (Th. Boon, Bruxelles); • Contrôle de l'expression génétique (M. Yaniv, Paris) : - Oncogènes mutants : cibles pour la thérapie (N. Lemoine, Londres); - Le couple prolifération-différenciation (L. Degos et H. de Thé, Paris) : - Nouvelles cibles et nouveaux concepts en chimiothérapie anti-cancéreuse (N.W. Lobbezoo, Amsterdam); - Chimioprévention des cancers (A. Costa, Milan); - Modulation de la résistance multidrogue associée à la $\mathrm{P}$-glycoprotéine des cellules tumorales (G. Atassi, Paris) ; - Chimio- et radioprotecteurs (M. Marty, Paris) ; - Transfert de gènes (Th. Velu, Bruxelles et T. Blankenstein, Berlin) ; - Anticorps monoclonaux humains (J. Banchereau, Lyon); - Conclusion (M. Boiron, Paris).

L'examen des posters et leur discussion auront lieu au moment de la pause-déjeuner.

3 posters seront sélectionnés pour présentation orale par le jury scientifique d'Eurocancer : P. Tambourin (Président), F. Calvo, H. Fridman (INSERM U.255), S. Gisselbrecht, C.-J. Larsen (INSERM U.301), A. Tavitian (INSERM U.301), A. Tavitian (INSERM U.248), Th. Tursz.

Le prix du meilleur poster sera remis en fin de journée par P. Tambourin et le comité scientifique.

Les actes de cette journée seront disponibles sur forme d'une co-édition INSERM/John Libbey Eurotext dans la collection des Colloques de I'INSERM. Pour renseignement complémentaire et inscription, s'adresser au secrétariat scientifique : M. Boiron, M. Marty, M.-C. Guédès, Centre Hayem, Hôpital Saint-Louis, 1, avenue Vellefaux, 75010 Paris. Tél. : 42.06.32.60 ou 42.03.36.56 - Télécopie : 42.41.14.70.

Un symposium international sur le thème "Ochratoxicose humaine et pathologies associées en Afrique et dans les pays en voie de développement " se tiendra à Bordeaux du 3 au 7 juillet 1993 avec le soutien de I'INSERM, de I'UNEP (United Nation Environment Program) et de la Région Aquitaine. II réunira les principaux spécialistes internationaux de l'ochratoxine $A$ (mycotoxine, contaminant alimentaire) (G. Dirheimer, H. Bartsch, M. Castegnaro, T. Kuiper-Goodman, P. Galtier, P. Bach, R. Plestina, B. Hald, P. Steyn, $Y$. Ueno, etc.) et des équipes de chercheurs ayant mis en évidence des maladies liées à l'ochratoxicose humaine et animale dans leurs pays respectifs (Afrique, Asie).

Les objectifs principaux sont les suivants :

- savoir si la néphropathie endémique des Balkans, vraisemblablement due

à l'ochratoxine $A$, existe en dehors de cette aire géographique ;

- quelles sont les pathologies associées à l'ochratoxicose dans les pays en voie de développement, en comparaison avec ce qui est observé dans les Balkans ;

- comment expliquer la spécificité rénale de cette mycotoxicose (néphrotoxicité et tumeurs) ;

- quels sont les moyens de prévention et/ou de traitement de l'ochratoxicose ( 25 à $60 \%$ au moins des personnes sont OTA-positives en Europe) ; - comment mettre en place un réseau Nord-Sud de collaboration sur ce sujet qui concerne aussi bien les pays industrialisés que les pays sousdéveloppés.

Les actes paraîtront pour le symposium, sous forme d'une co-édition INSERM/John Libbey Eurotext dans la collection des Colloques de I'INSERM.

Pour tout renseignement et inscription, s'adresser avant le 2 B février 1993 au Pr. E. E. Creppy, Laboratoire de Toxicologie, Université de Bordeaux II, 33076 Bordeaux cedex, "Tél. : 56.91.84.07"Télécopie : 56.91.14.16. 